\title{
Plasmocitoma testicular bilateral secundario. Aportación de un caso y revisión de la literatura
}

\author{
Garrido Abad P, Coloma Del Peso A, Bocardo Fajardo G, Jiménez Gálvez M, Herranz Fernández \\ LM, Arellano Gañán R, Pereira Sanz I, Reina Durán T*.
}

Servicio de Urología. *Servicio de Anatomía Patológica. Hospital de La Princesa. Madrid.

Actas Urol Esp. 2008;32(10):1039-1042

\section{RESUMEN}

\section{PLASMOCITOMA TESTICULAR BILATERAL SECUNDARIO. APORTACIÓN DE UN CASO Y REVISIÓN DE LA LITERATURA}

El plasmocitoma testicular es una neoplasia de células plasmáticas muy poco frecuente. Generalmente se trata de un hallazgo de autopsia carente de expresión clínica, aunque en algunas ocasiones puede constituir una manifestación de un mieloma múltiple y más excepcionalmente ser su única localización. La afectación bilateral es extremadamente infrecuente. Presentamos el caso de un varón de 58 años con mieloma múltiple en remisión que acude a consulta de urología por aumento progresivo del tamaño testicular bilateral. Tras realización de ecografía y PAAF testicular se diagnostica un plasmocitoma testicular bilateral. El tratamiento incluye cirugía (orquiectomía), radio y quimioterapia. El pronóstico de los pacientes depende de si se trata de un caso de plasmocitoma testicular solitario o con mieloma múltiple asociado.

Palabras clave: Tumor testicular. Plasmocitoma. Mieloma.

\section{ABSTRACT \\ SECONDARY BILATERAL TESTICULAR PLASMACYTOMA. CASE REPORT AND REVIEW OF THE LITERATURE}

Testicular plasmacytoma is a very unfrequent plasma cells neoplasm. It is usually an incidental autopsy finding devoid of clinical expression, but it presents sometimes as part of a multiple myeloma and is exceptionally its only location. Bilateral involvement is extremely unfrequent. We report the case of a 58 years old man diagnosed with multiple myeloma in complete remission who complains about progressive enlargement of both testicles. After ultrasonography and PAAF, bilateral testicular plasmacytoma is diagnosed. Treatment includes surgery (orchiectomy), radiotherapy and chemotherapy. Prognostic is worse when multiple myeloma is associated.

Keywords: Testicular tumour. Plasmacytoma. Myeloma.

$\mathrm{E}^{1}$ plasmocitoma es un tumor de células plasmáticas que afecta a la médula ósea o localizaciones extramedulares. El plasmocitoma testicular representa únicamente un $2 \%$ de todas las neoplasias de células plasmáticas, generalmente se trata de un hallazgo de autopsia carente de expresión clínica, aunque en algunas ocasiones puede constituir una manifestación de un mieloma múltiple (MM) y más excepcionalmente ser su única localización ${ }^{1}$.

El pronóstico del plasmacitoma testicular depende en gran medida de su asociación o no a un mie- loma múltiple. En este último caso, la mayoría de los pacientes presentan progresión de la enfermedad y mueren a consecuencia del tumor ${ }^{2,3}$.

El tratamiento incluye cirugía (orquiectomía), radio y/o quimioterapia, sin haber sido definida aún la terapia más adecuada.

\section{CASO CLÍnICO}

Presentamos el caso de un paciente varón de 58 años de edad diagnosticado de Mieloma Múltiple tipo Bence-Jones, estadio III-B, en el año 2003. Recibió 
entonces tratamiento con VAD (Vincristina, Adriamicina y Dexametasona), junto con bifosfonatos mensuales y soporte con Darbopoyetina semanal, alcanzando respuesta objetiva, tras lo cual se realizó transplante autólogo de sangre periférica (TASPE) y tratamiento con Melfalán 200. La evolución posttransplante fue buena, alcanzando remisión completa.

En el año 2006 el paciente comienza de nuevo con sintomatología y hallazgos exploratorios compatibles con progresión sistémica del MM. Es remitido a la consulta de urología debido a crecimiento progresivo e indoloro de ambos testículos, en mayor medida del lado izquierdo, en los últimos dos meses.

En la exploración física se aprecia teste izquierdo con hidrocele y engrosamiento duro e irregular que no permite distinguir estructuras con facilidad. El teste derecho también se encuentra discretamente aumentado de tamaño con consistencia aumentada, no encontrando alteraciones en el resto de estructuras genitales.

Dentro de las pruebas complementarias no encontramos modificaciones significativas. Los marcadores tumorales testiculares muestran unos valores normales: LDH: 231 UI (240-280); Alfa-fetoproteína: 2,3 ng/ml.; Beta-HCG: <1,20 mUI/ml.

Se realiza ecografía testicular que se informa como aumento de tamaño testicular izquierdo con un eje longitudinal de $55 \mathrm{~mm}$. frente a $36 \mathrm{~mm}$. del derecho. Ambos parénquimas testiculares tienen una estructura ecográfica muy heterogénea, con aspecto atigrado e irregular. En estudio doppler se observa importante aumento de la vascularización en ambos parénquimas testiculares. Los hallazgos son compatibles con infiltración testicular bilateral por células neoplásicas que debido a sus antecedentes sería con mayor probabilidad de tipo mielomatoso (Figs. 1, 2 y 3).

Se realiza punción-aspiración con aguja fina de testículo izquierdo mediante control ecográfico con diagnóstico anatomopatológico de plasmocitoma testicular (Fig. 4).

Además se realiza estudio de extensión donde se demuestran lesiones osteolíticas generalizadas.

Debido a ello, el paciente recibe tratamiento con radioterapia conformacional con planificación 3D de carácter radical sobre ambos testículos y se inicia nuevo tratamiento con quimioterapia sistémica (Velcade-Doxorrubicina liposomal).

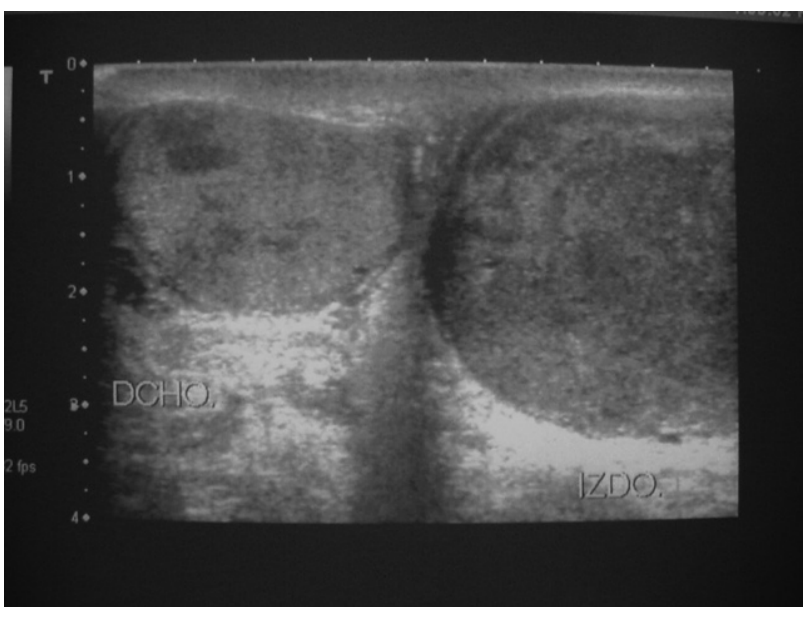

FIGURA 1. Imagen ecográfica de ambos testiculos.

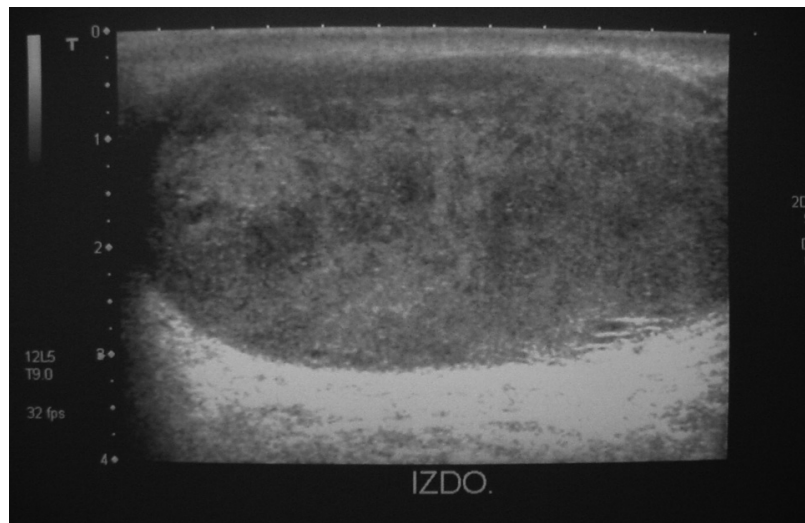

FIGURA 2. Imagen ecográfica de testiculo izquierdo.

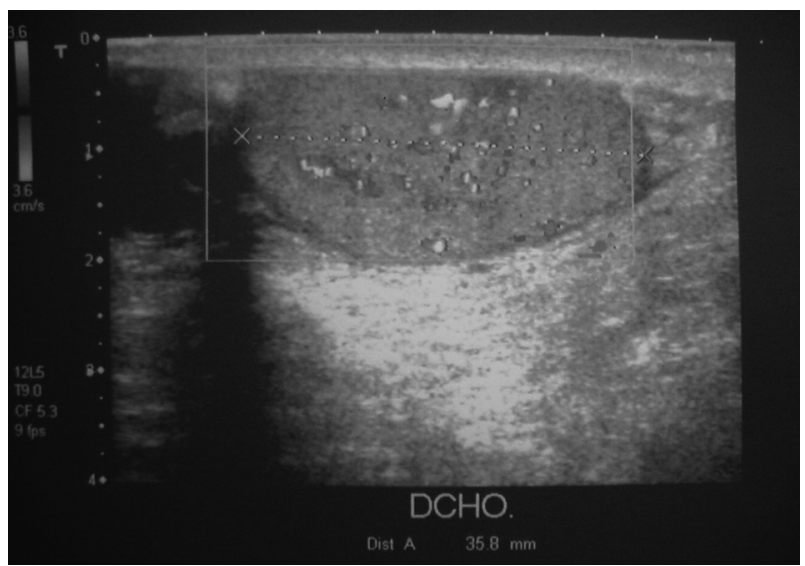

FIGURA 3. Imagen ecográfica de testiculo derecho.

\section{DISCUSION}

El mieloma múltiple es una enfermedad caracterizada por una producción y excreción aberrante de proteínas monoclonales homogéneas (electroforética e inmunológicamente) por un grupo de células plasmáticas neoplásicas. Este grupo celular prolifera 


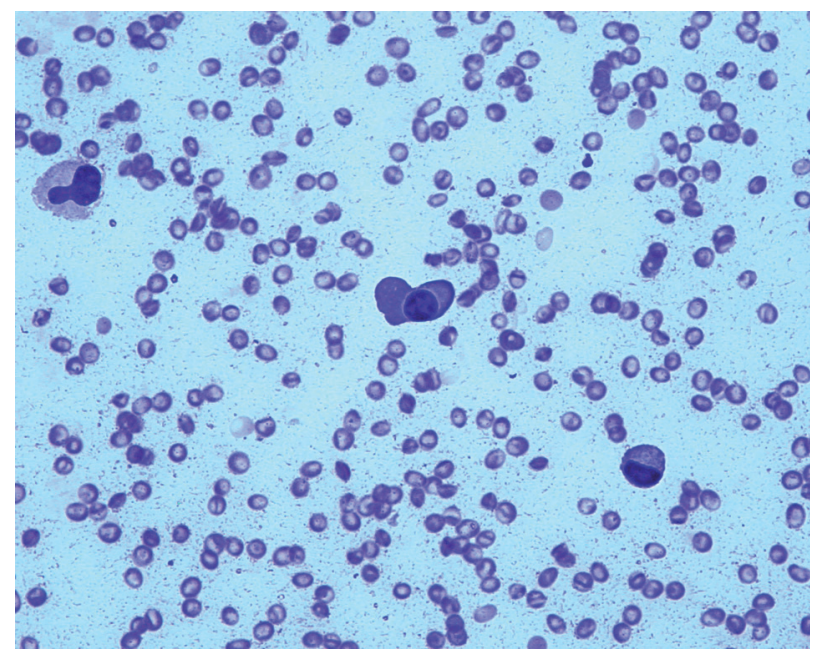

FIGURA 4. Imagen al microscopio (20x) de PAAF testicular donde se observan células plasmáticas irregulares con núcleos excéntricos y amplio citoplasma sobre fondo hemático.

originalmente en la médula ósea, invadiendo posteriormente el hueso adyacente con progresiva afectación esquelética generalizada.

Debido a unas condiciones favorables en algunas ocasiones las células plasmáticas neoplásicas se presentan en localizaciones extra-esqueléticas, pasando a denominarse entonces plasmocitoma extramedular ${ }^{4}$. En estudios en autopsias de pacientes con mieloma se ha objetivado afectación extramedular de hasta un 65-70\%5,6. Los plasmocitomas extramedulares que afectan al testículo son extremadamente raros, estimándose su incidencia en el $2 \%$ de todos los casos de neoplasias de células plasmáticas ${ }^{2}$. El plasmocitoma solitario es una entidad poco frecuente y representa únicamente el 6\% de todas las neoplasias de células plasmáticas ${ }^{7}$.

El plasmocitoma testicular presenta un rango de incidencia de entre el 0,03\% y el 0,1\% de todos los tumores primarios y secundarios que afectan al testículo. Entre los pacientes con mieloma múltiple, la incidencia de plasmocitoma que afecta al testículo es de entre un $0,6 \%$ y un $2,7 \%$ del total ${ }^{5,8}$. Levin y Mostofi revisaron en los años setenta 6.000 tumores testiculares con aparición de sólo 7 casos de mieloma testicular (aproximadamente 0,1\%) en el Armed Forces Institute of Pathology ${ }^{9}$. En raras ocasiones puede constituir la primera manifestación de un mieloma múltiple o, excepcionalmente, ser la localización única de una neoplasia de células plasmáticas.
Fue descrito por primera vez en 1939 y desde entonces menos de 60 casos han sido descritos en la literatura ${ }^{2,10,11}$ Se han descrito casos de plasmocitoma testicular en pacientes de entre 26 y 89 años, con una edad media de 56,5 años ${ }^{4}$. La afectación testicular bilateral por plasmocitoma es extremadamente rara, suele ocurrir de forma asincrónica y mas raramente de manera sincrónica. Hasta el momento actual únicamente 3 casos de afectación testicular bilateral sincrónica por plasmocitoma han sido publicados en la literatura ${ }^{12,13}$.

Las localizaciones más frecuentes de plasmocitomas extramedulares descritas son el tracto urinario superior, tejido linfoide submucoso de nasofaringe / senos paranasales, ganglios linfáticos, bazo, pulmón, riñón, glándula suprarrenal, páncreas, pleura, pericardio, músculo esquelético, mama y ovario ${ }^{5,14}$.

El plasmocitoma testicular se presenta como cualquier otra masa, primaria o metastásica, testicular. Afecta al parénquima testicular, aunque en ocasiones puede aparecer también en epidídimo. Se presenta con tumefacción indolora y en ocasiones se ha descrito con hidrocele asociado ${ }^{15}$. Histológicamente la infiltración se demuestra por la presencia de células plasmáticas atípicas. Mediante técnicas inmunohistoquímicas se objetiva expresión de inmunoglobulinas monotípicas citoplasmáticas.

El diagnóstico diferencial de las neoplasias de células plasmáticas del testículo hay que establecerlo fundamentalmente con el seminoma (particularmente su variante espermatocítica) y con los linfomas. El relativo monomorfismo celular, junto con la morfología plasmocelular, ausencia de crecimiento intratubular y estudio inmunohistoquímico (positividad para cadena ligera kappa y negatividad para la fosfatasa alcalina placentaria) permiten excluir la primera posibilidad. Las células de los linfomas habitualmente presentan menor citoplasma, carecen de halo paranuclear, con frecuencia se acompañan de esclerosis y el patrón inmunohistoquímico generalmente muestra positividad para el antígeno CD20 o CD23 y negatividad para el EMA10.

Debido a que la mayor parte de los pacientes con mieloma testicular se diagnostican en el contexto de una enfermedad sistémica, resulta obligado un estudio exhaustivo del paciente para descartar un mieloma múltiple o menos frecuentemente otros plasmocitomas ${ }^{3}$. Muchos autores piensan que, con 
tiempo suficiente, los pacientes con mieloma testicular desarrollarán mieloma extratesticular, si no lo presentan ya en el momento del diagnóstico ${ }^{12}$.

El pronóstico del plasmocitoma testicular depende en gran medida de su asociación o no a un mieloma múltiple, siguiendo una evolución mucho más desfavorable en los casos de asociación con éste último, con una supervivencia media de 12 meses $^{16}$. Pacientes con plasmocitomas extramedulares que no afectan a cabeza o cuello, tal y como es el caso del plasmocitoma testicular parecen presentar un pronóstico más desfavorable en $^{2,3,17}$.

En los pacientes con enfermedad diseminada se han aplicado una gran diversidad de tratamientos, que incluyen orquiectomía +/- quimioterapia +/radioterapia $^{18}$. En algún caso se ha utilizado también interferón dentro del tratamiento ${ }^{19}$.

En una extensa revisión de la literatura por Anghel et al. de todos los casos de plasmocitoma testicular con previa o simultánea presencia mieloma múltiple y/o plasmocitoma extramedular descritos hasta 2002 (34), la mayoría se trataron con orquiectomía, asociada o seguida de radioterapia, quimioterapia o ambas. La mayor parte de los pacientes tuvieron un pronóstico infausto, con progresión de la enfermedad y muerte derivada de la misma. Sólo 4 se encontraban libres de enfermedad, con un seguimiento medio de 12 meses $^{4}$.

En el caso del plasmocitoma testicular solitario el tratamiento es la orquiectomía radical, continuando aún en discusión la efectividad de tratamientos quimio o radioterápicos. Se han descrito casos de tratamiento exclusivo con orquiectomía sin aparición de recurrencia durante cortos periodos de seguimiento $^{12,18}$.

\section{REFERENCIAS}

1. Álvarez-Múgica M, Jalón Monzón A, Bulnes Vázquez V, Aguilar Andrea C, Fernández Gómez JM, González Álvarez RC, et al. Secondary Testicular Plasmocytoma. Arch Esp Urol. 2007;60(1): 99-102.

2. Ulbright TM, Amin MB, Young RH. Miscellaneous primary tumors of the testis, adnexa, and spermatic cord, hematopoietic tumors and secondary tumors. In: Rosai J, ed. Atlas of tumor pathology, Tumors of the testis, adnexa, spermatic cord, and scrotum, tirad series. Washington DC: Armed Forces Institute of Pathology; 1999: 235-90.
3. Ferry JA, Young RH, Scully RE. Testicular and epididymal plasmacytoma: a report of 7 cases, including three that were the initial manifestation of plasma cell myeloma. Am J Surg Pathol. 1997;21(5):590-598.

4. Anghel G, Petti N, Remotti D, Rucio C, Blandino F, Majolino I. Testicular Plasmacytoma: Report of a case and Review of the Literature. Am J Hem. 2002;71(2):98-104.

5. Hou TY, Dai MS, Kao WY. Testicular plasmacytoma with bone dissemination without medullary plasmacytosis. Ann Hematol. 2003;82(8):518-520.

6. Pasmantier MW, Azar HA. Extraskeletal spread in múltiple plasma cell myeloma. Cancer. 1969;23(1):167-174.

7. Knowling MA, Harwood AR, Bergsagel DE. Comparison of extramedullary plasmacytomas with solitary and multiple plasma cell tumors of bone. J Clin Oncol. 1983;1(4):255-262.

8. Chica G, Jonson DE, Ayala AG. Plasmacytoma of testis presenting as primary testicular tumor. Urology 1978;11:90. Hayes DW, Bennett WA, Heck FJ. Extramedullary lesions in multiple mieloma. Arch Pathol (Chicago) 1953;53:262.

9. Levin HS, Mastofi FK. Symptomatic plasmacytoma of the testis. Cancer. 1970;25(5):1193-1203.

10. García Escudero A, Segura-Sánchez J, Navarro Bustos G, Poyato Galán JM, Figueredo Manrique A, González Cámpora R. Plasmocitoma testicular: descripción de un caso. Rev Esp Patol. 2003;36(3):329-332.

11. Ulrich H. Múltiple myeloma. Arch Intern Med 1939; 64:994.

12. Pham TH, Shetty SD, Stone $\mathrm{CH}$, De Peralta-Venturina M, Menon M. Bilateral synchronous testicular plasmacytoma. J Urol. 2000;164(3 Pt 1):781. Levin HS, Mostofi FK. Symptomatic plasmacytoma of the testis. Cancer. 1970;25(5):1193-1203.

13. Castagna M, Gaeta P, Cecchi M, Pagni GL, Pingitore R. Bilateral synchronous testicular involvement in múltiple myeloma. Case report and review of the literature. Tumori. 1997;83(4):768771.

14. Gordon AJ, Churg J. Visceral involvement in múltiple myeloma. N Y State J Med. 1949;49(3):282.

15. Tomoko S, Takashi K, Torhu A. A case of extramedullary plasmacytoma of the testis diagnosed cystologically by scrotocentesis. J Jpn Soc Clin Cytol. 1995;34: 1156-9.

16. Oppenheim P, Cohen S, Anders K. Testicular plasmacytoma. A case report with immunohistochemical studies and literature review. Arch pathol Lab Med. 1991;115(6):629-632.

17. White J, Chan YF. Solitary testicular plasmacytoma. Br J Urol. 1995;75(1):107-108.

18. Shafqat A, Yum MN, Abanour R, Ganjoo KN. Unusual Locations of Involvement by malignancies. Case1. Testicular plasmacytoma. J Clin Oncol. 2003;21(17):3368-3369.

19. Brambilla G, Pisoni A, Ballarti E, Turri E, Zaroli A, Montalbetti L. Plasmacitoma del testicolo. Descrizione di un caso clinico e revisione della letteratura. Minerva Med. 1993;84(4):211-215.

Correspondencia autor: Dr. P. Garrido Abad

Servicio de Urología. Hospital Universitario de La Princesa Diego de León, 62 - 28006 Madrid

Tel.: 915202200

E-mail autor: pgabad@hotmail.com

Información artículo: Nota clínica

Trabajo recibido: marzo 2007

Trabajo aceptado: abril 2007 